\title{
Automated Car Parking System
}

\author{
Syed Asif Ali \\ Sindh Madressatul Islam University \\ Karachi, Pakistan
}

\author{
Syeda Wajiha Naim \\ Sindh Madressatul Islam University \\ Karachi, Pakistan
}

\author{
Basit Hassan Qureshi \\ Sindh Madressatul Islam University \\ Karachi, Pakistan
}

\author{
Abdul Sattar \\ Sindh Madressatul Islam University, \\ Karachi, Pakistan
}

\author{
M. Fahad \\ Sindh Madressatul Islam University \\ Karachi, Pakistan
}

\begin{abstract}
Pakistan is one of the over-populated countries in world and maximum number of people prefer using their own vehicles as conveyance. Since roads in Pakistan are overloaded with different types of vehicles, people usually face parking problem in parking cars and other transport on regular basis. Parking has now become a major issue of the country. This paper is an attempt to propose a design of automated car parking system directed by an android application, that maintains the number of cars to be parked on designated area by automating the parking $\&$ un-parking of the vehicles with the help of instructions of an android application. This automated car parking system aims to reduce involvement of humans in directing people to park their vehicles by mechanizing this process. This system would be very useful in reducing the time required for searching the available parking space within parking area.
\end{abstract}

\section{Keywords}

Automated car parking system, Android Application, Image Processing, Raspberry PI3 Module

\section{INTRODUCTION}

People mostly opt to visit various public places like Shopping malls, 5-star and 7-star hotels, multiplex cinema halls, etc for recreation. The difficulty they encounter at these places is finding the available parking space. Most of the times they need to navigate through multiple parking slots to look for a free space for parking. In past buses and trains were the most popular means of commute among. The number of people migrating from rural areas to urban ones have drastically increased as they tend to move in metropolitans for better employment opportunities and other needs. The transport on roads have multiplied and there are more buses, trucks, motorbikes, rickshaws etc. on the streets and roads. Owing to the increase in use of vehicles, the parking has become a tiring, difficult and complicated taks. When people do not find slot for parking, they usually park their vehicles on busy roads that result in blocking the smooth flow of traffic. At present, in Pakistan, there is no planning in the development of parking areas hence deficiency in devising a systematic approach for parking system. There is a slight change, that the new building that are being developed plan slots of parking in their maps, but this also is not the solution of the problem as number of vehicles outnumber the available slots for parking in buildings. The manual control is there in some areas but it is not so sound to accommodate the large number of vehicles.. The administration have implemented many techniques to ensure the ease of parking at car parking zones.
Mostly the people are not aware of the empty space at the parking areas. In proposed idea, the camera can be used to sense the empty space through Video Image Detection. The image can be captured and allowed to Image Segmentation and Edge Detection through boundaries with canny operator method. The moving car is to be parked at specific zone, at first the parking area needs to be identified at the zone and after that it is required to ascertain the empty parking space. In The parking area the Image Processing Technique can be implemented that will undergo the Image Segmentation and Edge Detection in addition to that a Counter will be introduced to count the Number of Entries/ Exit and it will be noted. This research is an attempt to find the variation between the moving image and the Stationary Image on the Basis of Variance in Brightness of the Image. The Existing process attempts on taking Time Differential Image. The problem is when the object is moving at high speed it is a tedious job to take snap of that image. The current work attempts to identify the specific area in parking lots by the respective number and the Sensor present at that lot. The Sensors can be placed at the parking area if the signal from transmitter will not be received by the sensor, then the sensor is sensed and result shows that the parking lot is filled.

\section{LITERATURE REVIEW}

In this research paper (Jawad A. et al.) proposed a system in which they illustrate the counter for the car entering the parking area and give instructions to the parking gate to not to be opened more cars when there are maximum number of cars. The same counter is made for the exit gate in which the number of cars that exit from the park are counted in reverse. The parking is operated through the piezoelectric sensor that depends on the weight of car (by pushing a small button) when button is pushed the counter adds and subtracts 1 from the counter number [1].

In another research paper, a system is proposed to maintain a record of the time; vehicles check in and check out. There are different pricing system for both; thr two wheelers and the four wheelers. The system will have two stand by PCs that will maintain the record of vehicles entering and exiting. Time management and control of vehicle is done by number plates recognition. The system will track whether the parking lot is full or not by tracking the entrance and exit of cars. The cost of the vehicles will be according to the time consumed by the vehicle [2].

A crowd source approach is suggested to be used by Huzefa Dargahwala in his paper, This tactic identifies parking slots/spots in streets and curbs where the risk of vehicles been taken away is low. The user will mark the slots/spots that will identify the genuine and fake slots/spots. The system will also 
highlight slots/spots that are close and are free alternatives to the paid ones. It will be a GPS based parking system that will use Google API for locating the parking slots/spots that are free and near. The data received from the users will help us know the interesting patterns in the most requested parking slots/spots [3].

Thus, we aim to propose a car parking system that represents a fully automated model with minimum human intervention and overcome the limitations of existing systems.

\section{PROPOSED SYSTEM}

In this project or prototype an intelligent car parking system will be developed based on the research done for the purpose. This system will be based on the digital image processing or image processing.

\section{METHODOLOGY}

The system will be implemented in three modules.

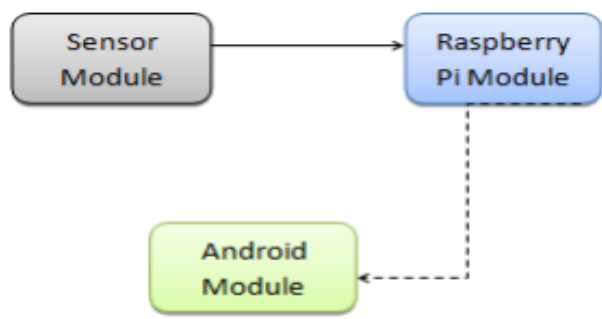

Figure 1: Block Systematic Diagram of Proposed System

\subsection{Sensor Module}

This installation of this module will be made in the parking place. There will be a sensor node for each parking space. The IR- sensors will be used for the purpose.

\subsection{Raspberry Pi Module}

This module will be connected to the internet and will have connections from all sensor nodes.

\subsection{Android Module}

This module will be installed as an android application in the user phone. And will display the parking slot status.

\section{SYSTEM ARCHITECTURE}

A Raspberry Pi with various IR sensors attached to it will be required. The parking status will be determined by the IRsensors. Raspbian is the operating system of the raspberry Pi and Android app will be used to identify the status of the parking in the parking lot. Wi-Fi network will be required to access the parking lot setup by android app. Users will be able to check the parking status on their cell phones. The Raspberry Pi interfaced with the IR sensors to determine the parking status will be the hardware setup of the project. Hence the raspberry pi becomes the hardware module of the system.

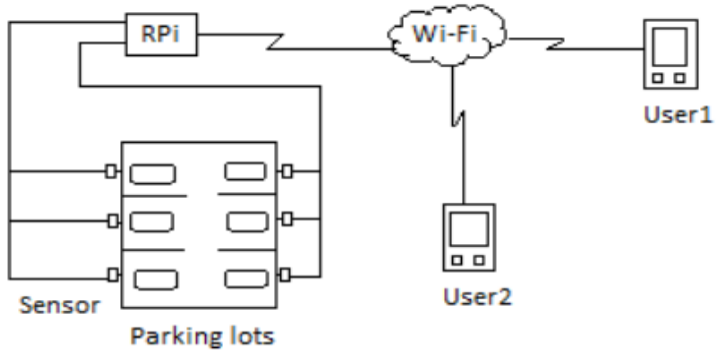

Figure 2: System Architecture

\section{CONCLUSION AND FUTURE SCOPE}

In this paper, the implementation of an Automated Car Parking system directed by Android Application is successfully examined. All the components used for the implementation of the system give efficient output at different stages of implementation. The interfaces set between different components give an effective communication to overall working of the system. Thus, the system functioning is effective and is recommended for commercial implementation. Vehicle parking can be made easier if the proposed architecture is implemented to daily life. An Image processing technique can be employed for the process of identification of the parking area and the number of empty parking slots. The parking slots can be easily identified and the shape of that particular slot can be determined which results in occurrence of parking the vehicle in safe area within short span of time without any delay.

\section{REFERENCES}

[1] M. F. H. Z. H. M. Ali Abd Al-Zahra Jawad, "Design and Implementation of Smart Car Parking System," Researchgate, Hillah, 2016.

[2] B. KHANAL, "Vehicle Parking Management System," New Delhi, 2015.

[3] A. G. Z. B. N. M. ,. Huzefa Dargahwala, "GPS Based Parking System for Unplanned Metrocities with Visualization of Request Data.," International Journal of Engineering Research \& Technology (IJERT), p. 4, 2013.

[4] Bagula, Antoine, Lorenzo Castelli, and Marco Zennaro. On The Design of Smart Parking Networks in the Smart Cities: An Optimal Sensor Placement Model. Open Access Sensors 15 (2015): 15443-15467.

[5] Fahmy, Hossam M. A. Wireless Sensor Networks: Concepts, Applications, Experimentation and Analysis. , 2016.

[6] Joseph J. Patil R. G. Narahari S. K. K. Didagi Y. Bapat J. "Wireless Sensor Network Based Smart Parking System". 